\title{
Virtual Time-Variant Model of the Eustachian Tube
}

\author{
Christiane Antweiler and Peter Vary \\ Institute of Communication Systems and Data Processing \\ RWTH Aachen University \\ Aachen, Germany \\ Email: antweiler@ind.rwth-aachen.de
}

\author{
Ercole Di Martino \\ Clinic for ENT Diseases and Plastic Head and Neck Surgery \\ Ev. Diakonie-Krankenhaus \\ Bremen, Germany \\ Email: e.dimartino@diako-bremen.de
}

\begin{abstract}
In contrast to most of the clinical examinations, the sonotubometric assessment of the Eustachian tube function allows the registration of the tube opening at the perforated, as well as at the intact eardrum in physiological conditions. In this paper a new procedure is introduced which performs a measurement of the transmission path nose/ear. With digital signal processing algorithms the generation of a new feature relevant for medical diagnostics is made accessible. The method is based on techniques known from speech processing: the acoustic tube model, the normalized least-mean-square (NLMS) algorithm with so-called perfect sequence (PSEQ) [1],[2] excitation and the LevinsonDurbin algorithm. Within the framework of an interdisciplinary cooperation we developed a new real-time acoustic measurement prototype which opens up entirely new possibilities in otological diagnostics.
\end{abstract}

\section{INTRODUCTION}

The Eustachian tube is responsible for ventilation, pressure equilibration and drainage of the middle ear and the mastoid. Since the physiology and the impact of the alterations of the Eustachian tube on the pathegenesis of middle ear diseases are not completely understood, numerous methods such as tympanometric, manometric, radiologic, endoscopic and sonotubometric approaches have been developed for the evaluation of the Eustachian tube function [3], [4], [5].

Sonotubometry is a method that allows the examination of the Eustachian tube under physiological conditions. For this purpose an acoustic signal (e.g. $8 \mathrm{kHz}$ sine) is applied via a loudspeaker in the nose and synchronously recorded with a microphone in the ear. The fluctuations of the sound intensity provoked by a tube opening manoeuvre such as yawning or swallowing indicate the activity of the Eustachian tube such as depicted in Fig. 1. One problem of this method is its poor quality and unreliability of the measurements.
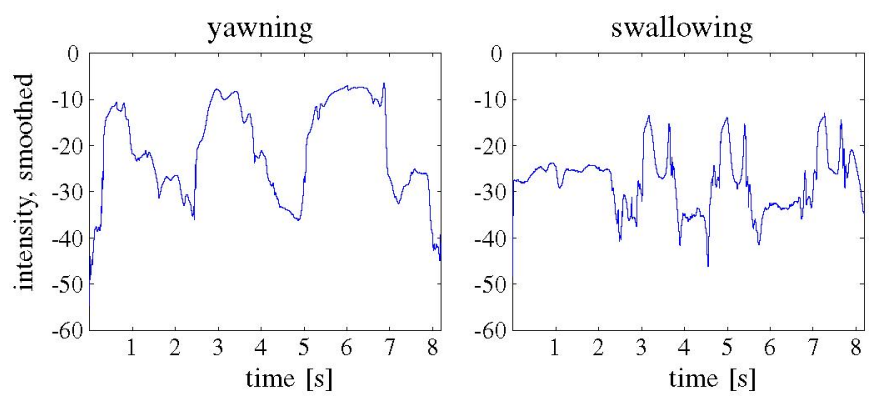

Fig. 1. Results of the sonotubometric approach with $8 \mathrm{kHz}$ sinus
In Sec. II we introduce a novel approach where the transmission link between the nose and the ear is excited with so-called perfect sequences (PSEQ) [1], [2]. The subsequent system identification [7] results in impulse responses and frequency responses at each time instant. Besides the information at $8 \mathrm{kHz}$ we now obtain the information of the complete spectrum, whereas the effort of measurement remains the same. In Sec. III we outline to which extent this gain of information can be used to improve the diagnostic system. The results of preliminary clinical trials with twenty test persons corroborate the system-theoretical results (Sec. IV). We show that the additional information can be used to significantly improve clinical diagnostics. Finally, in Sec. V a brand new approach is introduced, where the dynamic Eustachian tube function is visualized with a virtual tube model.

\section{Acoustic Measurement System with PSEQs}

In the approach presented here, the $8 \mathrm{kHz}$ sinus tone commonly used in sonotubometry is replaced according to Fig. 2 by a perfect sequence (PSEQ) [1], [2]; a periodically repeated pseudo noise signal $p(k)$ of length $N$. The reaction of the nose/ear system $y(k)$ is recorded simultaneously at a sample rate of $f_{a}=32 \mathrm{kHz}$. Due to the fact that, technically, the Eustachian tube can be considered as a linear transmission system, a system identification based on the signals $p(k)$ and $y(k)$ is carried out.

PSEQs are characterized by their periodic auto-correlation function which vanishes for all out-of-phase values. With this property an NLMS-driven adaptation process [6] thus provides

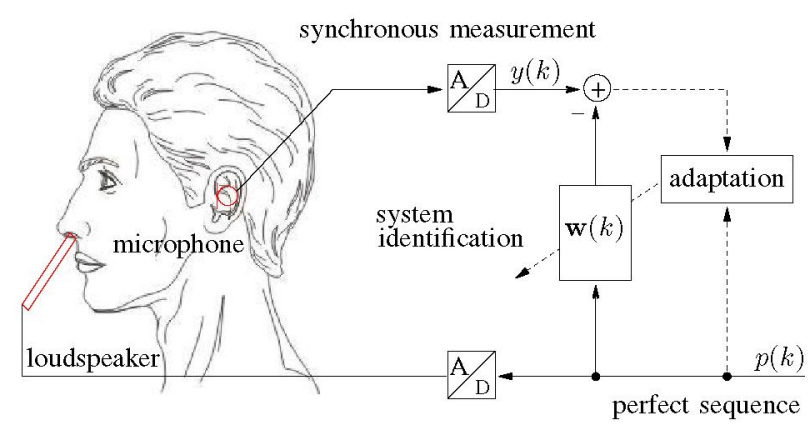

Fig. 2. Real-time measurement system with PSEQs 

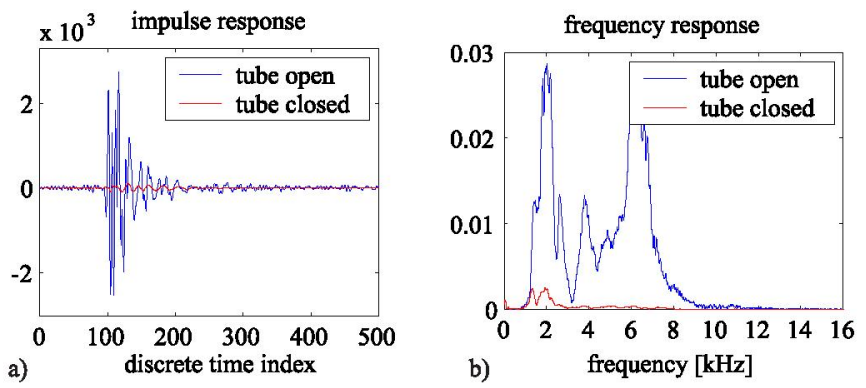

Fig. 3. Impulse- and frequency response in case of an open and closed tube

a complete identification of the nose/ear path in form of timevariant impulse responses $\mathbf{w}(k)$ [7].

Thus, rather than providing only one single amplitude value as does the conventional procedure, a complete impulse response $\mathbf{w}(k)$ of length $N$ is obtained. The impulse responses can then be transformed into frequency responses such that the entire frequency spectrum can be used for diagnostics. Figure 3 shows an example of two impulse responses and frequency responses recorded at two time instants; one with an open tube and one with a closed tube. Clearly, the differences in scale and form can be observed.

The novelty of this approach lies in the use of the new excitation signal $p(k)$ for this type of acoustic measurement and its application in medical tube function examinations. In comparison to the conventional measurement with sinus signals, additional information is obtained at the same measurement expenditure. The additional information can now be used to map the dynamic behaviour of the tube function in much better quality.

For this purpose, the norm of the impulse response $\mathbf{w}(k)$ is computed according to

$$
\Theta(k)=\|\mathbf{w}(k)\|^{2}=\sum_{i=0}^{N-1} w_{i}^{2}(k),
$$

which refers to the energy of the impulse response. The amplitude value used in sonotubometry indicates the intensity with which an $8 \mathrm{kHz}$ sinus tone is transmitted from the nose to the ear. The norm $\Theta(k)$ provides similar information with the energy of the individual impulse responses. However, in contrast to the conventional approach $\Theta(k)$ comprises the information of all the frequency components up to $16 \mathrm{kHz}$.

Apart from the possibilities for a system identification based on the NLMS algorithm, the PSEQs facilitate a highly efficient calculation of $\Theta(k)$. System-theoretically, it can be shown that

$$
\Theta(k)=\|\mathbf{w}(k)\|^{2}=\frac{\sum_{i=0}^{N-1} y^{2}(k-i)}{\sum_{i=0}^{N-1} p^{2}(k-i)}=\frac{1}{E_{p}} \sum_{i=0}^{N-1} y^{2}(k-i)
$$

holds in the case of PSEQs; where $E_{p}$ denotes the constant energy of the PSEQ. This allows an efficient computation of
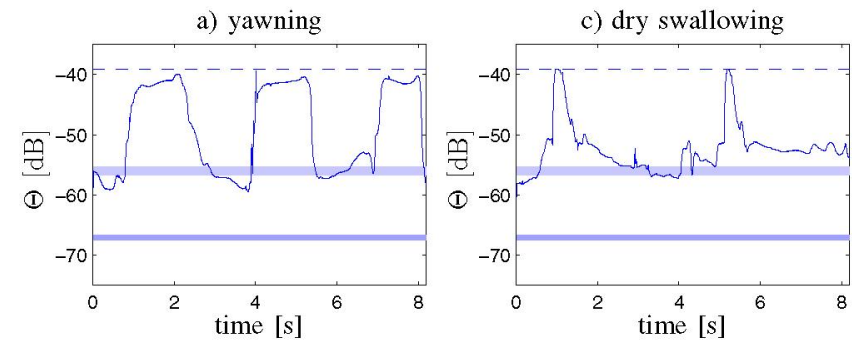

b) Valsalva
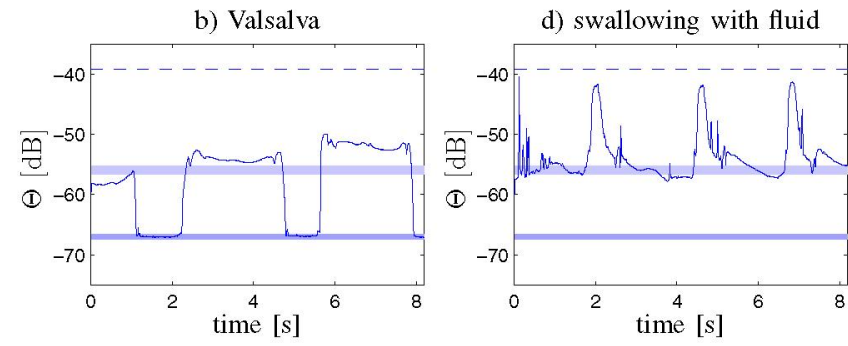

Fig. 4. Norm $\Theta(k)$ for four different manoeuvres

the norm $\Theta(k)$ even without explicit knowledge of the impulse responses $\mathbf{w}(k)$, i.e. as far as the norm $\Theta(k)$ is concerned, the system identification itself need not be performed.

Figure 4 comprises the results of the norm $\Theta(k)$ for one test person for different manoeuvres. For provocation of a tube opening manoeuvres such as yawning, dry and water swallowing as well as Valsalva were performed. Additionally, Fig. 4 includes some constant lines which characterize the

- quiet level (lower line, no PSEQ applied),

- passive level (mid line, with PSEQ, no manoeuvre) and

- peak level (dashed line, maximum over all curves).

Obviously, a valid norm curve must be located between the passive and the peak level. Fig. 4-a,c,d show typical results and are principally comparable to the results in Fig. 1. In the example of Fig. 4-b the Valsalva manoeuvre is susceptible for a failure of the sonotubometric measurement since the nostrils with the inserted tube have to be compressed to generate sufficient pressure to provoke a tube opening.

As pointed out before all the frequencies components up to $16 \mathrm{kHz}$ are reflected in the norm $\Theta(k)$. In the following section we will outline to which amount this gain of information can be exploited to improve the quality of the results.

\section{GAIN OF INFORMATION}

The examination of different spectra reveals that not all frequency responses of the test persons showed significant frequency components at $8 \mathrm{kHz}$, i.e. the transmission behaviour of the nose/ear system is potentially insufficient for this frequency range; see Fig. 5-a. As a result, the conventional approach with sinus signals may lead to non-interpretable sound intensity curves in spite of clear tube openings (Fig. 5-b), which is the reason for its poor quality and unreliability.

As the norm $\Theta(k)$ of the impulse responses $\mathbf{w}(k)$ exploits the information of all frequency components up to $16 \mathrm{kHz}$, an 


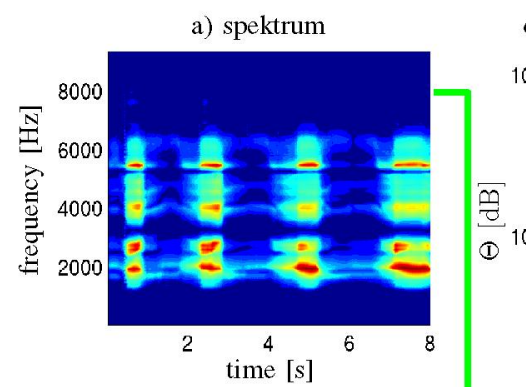

c) measurement with PSEQ

b) measurement with $8 \mathrm{kHz}$ sinus
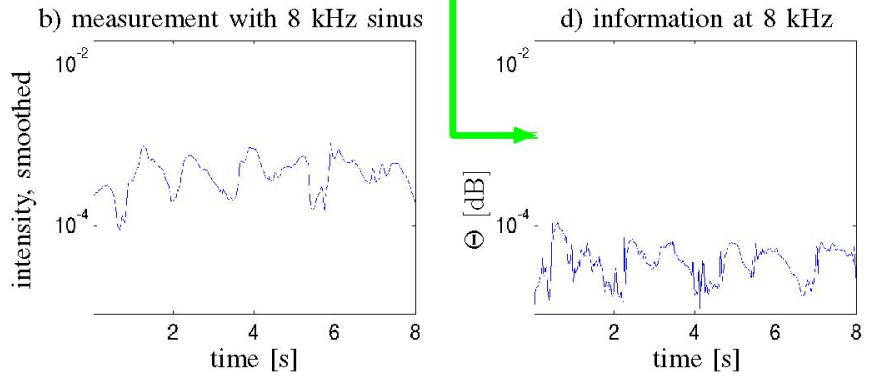

Fig. 5. Measurement with PSEQ (a,c,d) and $8 \mathrm{kHz}$ sinus (b)

improved representation of the dynamic tube activity according to Fig. 5-c can be observed.

The improvement due to the gain of information can be explained by an experiment. We extract the information at $8 \mathrm{kHz}$ out of the complete spectrum via a small notch filter and plot the corresponding norm $\Theta(k)$ in Fig. 5-d. As a result the curves in Fig. 5-b,d are based on the same extent of information, which is revealed in the similarity of the curves. Note that the curves were measured under the same conditions, but at different points of time. As these curves are based on limited information, tube openings cannot clearly be detected.

A comparison to Fig. 5-c indicates the significant improvement of the curve due to the gain of information.

\section{Clinical Examinations}

A prototype of the acoustic measurement system with PSEQs was applied successfully in combination with a recently developed analysis procedure in clinical examinations. A total of 320 comparative measurements using sinus signals and PSEQs were carried out with 20 test persons. A manual evaluation of the resulting curves revealed that $46 \%$ of the measurements with PSEQs were rated better than those with sinus signals. In $18.6 \%$ of the measurements, a tube opening could only be detected by means of PSEQs. The method applied here facilitates a real-time monitoring of the dynamics of Eustachian tube functioning under physiological conditions. With this concept, a significant improvement of the curves was achieved, from which clinical diagnostics will directly benefit. A further goal of the method is the extraction of new features from the spectrum, which are relevant in clinical diagnosis. One new and rather promising approach will be introduced in the next section.

\section{Visualisation of The Dynamic Eustachian Tube FUNCTION With A ViRTUAL MODELL}

So far medical diagnostics has mainly focused on the intensity with which an acoustic signal is transmitted from the nose to the ear. In this section, we present an entirely new method which extracts new features from the additional information in the form of impulse responses and frequency responses as described in Section II.

Many of the algorithms in digital speech processing are based on the model of speech production. Acoustic and physical observations can be used to derive a digital model of the process of speech production [8] [9]. It basically consists of two components; stimulation (air stream) and vocal tract filter, both of which are directly involved in signal production and signal form. The fact that most current speech coding algorithms and standards are based on this digital model of speech production illustrates the particular relevance of this approach. The digital speech tract filter can be derived from the tube model, as it is called. For this purpose, the human vocal tract is approximated by several cylindrical lossless tube segments with varying cross-sectional areas. The corresponding signal flow diagram solely contains sum, multiply and delay operators, and can thus be directly converted into a timediscrete model.

The principle of modelling the vocal tract by different tube segments and its substitution via a digital filter can, conversely, be transferred to the nose/ear transmission system. In a first key experiment, a simple virtual model of the nose/ear transmission system was derived in analogy to the tube model of the vocal tract. Time-variant cross sections of a virtual tube model were determined by means of the measured impulse responses of the test persons. The principle is shown in Fig. 6.

Based on the impulse responses $w$, the auto-correlation coefficients $\varphi_{w w}$ have to be calculated for each time instant, and the normal equation system must be set up. The equation system is then solved, e.g., by means of the Levinson-Durbin algorithm [10]. In analogy to the tube model used in speech processing, the resulting reflection coefficients $K$ are transformed iteratively into cross-sectional areas $A$ according to

$$
A_{i-1}=A_{i} \frac{1+K_{i}}{1-K_{i}} .
$$

The parameters of the model must be adapted to our special application. The prediction degree $n$, the final reflection

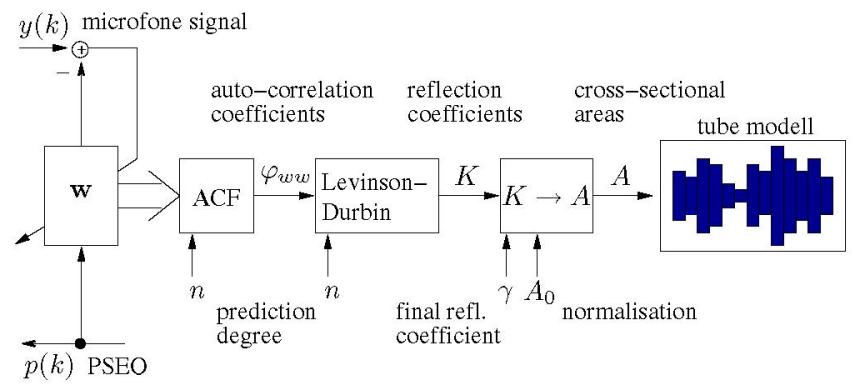

Fig. 6. Principle of the transformation into a virtual tube model 

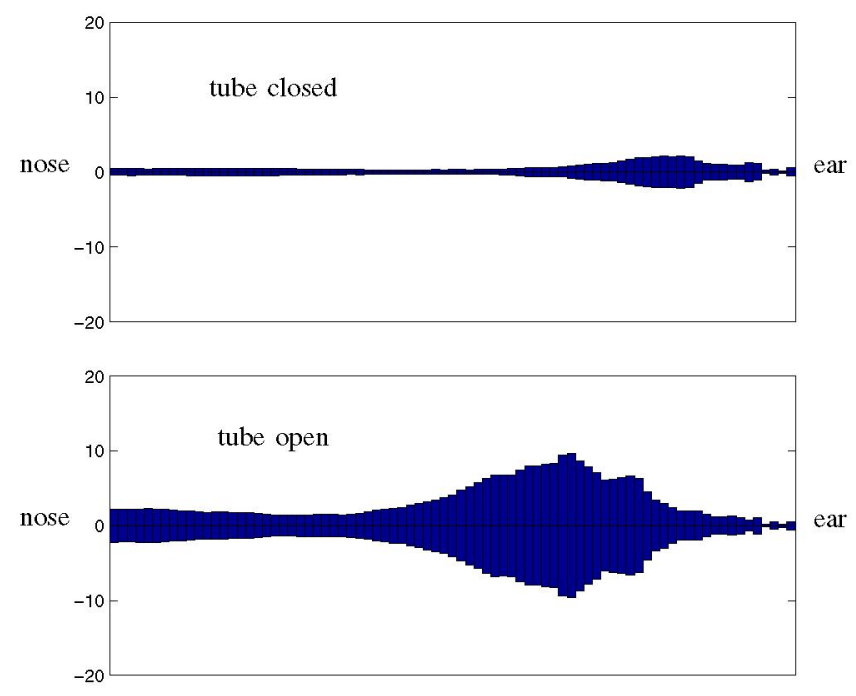

Fig. 7. Example of the virtual tube model for two time instants

coefficient at the ear $\gamma$, and the normalisation factor $A_{0}$ are particularly decisive for the shape of the model. The structure of the model can be improved by an appropriate choice of these parameters.

In Fig. 7, the typical structures of the virtual model of both the open and the closed Eustachian tube are illustrated by two examples.

The computation and graphic representation of a virtual time-variant model of the nose/ear transmission system is achieved by means of a prototype. The dynamic opening and closing process of the tube is visualised by an animation of the virtual tube model over time. By means of this model, a kind of "acoustic tube endoscopy" can be carried out.

So far medical diagnostics has mainly focused on the sound intensity with which a sinus signal of $8 \mathrm{kHz}$ is transmitted from the nose to the ear. In the norm the energy of the impulse response is considered. Compared to the conventional sonotubometry approach this leads to significantly improved results. The virtual tube model, however, is independent from the energy of the impulse response. Instead, the model is solely based on the analysis of the spectral composition of the transmission function. In this way, a completely complementary information is exploited. A new feature relevant for medical diagnostics is made accessible and thus new insights into the dynamics of the Eustachian tube function in physiological conditions can be gained.

\section{CONCLUSION}

Within the framework of an interdisciplinary cooperation a new real-time acoustic measurement prototype for medical diagnostics was developed.

In the novel approach the transmission link between the nose and the ear is technically considered as a linear transmission system. A system identification is performed applying PSEQs as the excitation signal. Thus, rather than providing only one single amplitude value as does the conventional sonotubometry applying a sinus signal, here a complete impulse response is obtained. As a result, additional information is obtained at the same measurement expenditure.

This gain of information is utilized on one handside to map the dynamic behavior of the tube function in much better quality by means of the norm of the impulse response. As the transmission behavior of the nose/ear system is potentially insufficient in the range of $8 \mathrm{kHz}$ the conventional approach may lead to non-interpretable sound intensity curves in spite of clear tube openings. Due to the fact that the norm of the impulse response exploits the information of all frequency components up to $16 \mathrm{kHz}$, a significant improvement in the visualisation of the Eustachian tube activity can be achieved.

First clinical studies have proved the theoretical results. A total of 320 measurements were carried out with 20 test persons. In almost $50 \%$ of the measurements with PSEQs the results were rated better than those with sinus signals. In almost $20 \%$ of the measurements a tube opening could only be detected by means of PSEQs.

On the other handside the information gain was exploited by the extraction of a completely new feature which takes the form of the frequency response into account. In a first key experiment a simple virtual tube model for the nose/ear transmission link was derived in analogy to the tube model of the vocal tract filter. With an animation of the virtual tube model over time the dynamic open and closing process of the Eustachian tube can be visualized. As a result the proposed method allows a real-time monitoring of the Eustachian tube activity under physiological conditions and can be interpreted as a kind of "acoustic tube endoscopy". Future work will aim at the improvement of the correlation between virtual tube model and anatomy.

It is of special interest that the norm and the virtual tube model are based on orthogonal information such that the features relevant for diagostics complement each other. It opens up entirely new possibilities in otological diagnostics.

\section{REFERENCES}

[1] H. D. Lüke, Korrelationssignale, Springer, Berlin, 1992.

[2] H. D. Lüke, H. D. Schotten, "Odd-perfect, almost binary correlation sequences", IEEE Trans. Aerospace a. Electron. Syst. AES-31, 1995.

[3] I. Honjo, "Evaluation of static and dynamic functions of the eustachian tube", in The Eustachian tube in middle ear diseases, Springer, Tokyo, pp. $25-38,1988$.

[4] T. P. McBride, C. Dekray, M. Cunningham M., W. Doyle, "Evaluation of noninvasive Eustachian tube function tests in normal adults", Laryngoscope, Vol. 98, pp. 655-658, 1988.

[5] E. Di Martino, R. Thaden, G. A. Krombach, M. Westhofen, "Eustachian tube function tests. Current knowledge", HNO, Vol. 52, pp. 1029-1040, 2004.

[6] S. Haykin, Adaptive Filter Theory, Prentice Hall, New Jersey 07632, 1986.

[7] C. Antweiler, M. Antweiler, "System Identification with Perfect Sequences Based on the NLMS Algorithm", $A E \ddot{U}$, Vol. 49, No. 3, pp. 129$134,1995$.

[8] P. Vary, U. Heute, W. Hess, Digitale Sprachsignalverarbeitung B. G. Teubner, Stuttgart, 1998

[9] T. Parsons, Voice and Speech Processing. McGraw-Hill, New-York, 1987.

[10] J. Makhoul, "Linear Prediction: A Tutorial Review", IEEE Proceedings, vol. 63, April, pp. 561-580. 